\title{
An adult female with headache and bilateral shank ache
}

\author{
Ewa Cyrańska-Chyrek, Ewelina Szczepanek-Parulska, Anna Ostałowska, Patrycja Antosik, \\ Rafael Jedliński, Marek Ruchała \\ Department of Endocrinology, Metabolism, and Internal Medicine, Poznan University of Medical Sciences, Poznan, Poland
}

Key words: Paget's disease; shank ache; alkaline phosphatase

\section{Case presentation}

Herewith we report a 54-year-old woman with a history of ovarian cancer, diagnosed 11 years before and treated surgically followed by chemotherapy. She also suffered from hypertension treated with bisoprolol ( $5 \mathrm{mg} /$ day) for the past five years. During oncological follow-up, nine years after surgery, on abdominal CT she was diagnosed with bilateral adrenal tumours. On admission her main complaint was headache and bilateral shank ache of six months' duration.

She was eventually diagnosed with non-functional bilateral adrenal tumours, being benign lipid-rich adenomas ( $21 \mathrm{~mm}$ and density of 7 Hounsfield Units [HU], and $10 \mathrm{~mm}$ and density of $3 \mathrm{HU}$ ). In addition, she presented with slightly increased parathormone level with normocalcaemia. No detectable parathyroid adenoma was seen on ultrasound examination or on MIBI scintiscan. Increased alkaline phosphatase and suboptimal concentration of vitamin D were detected (Tab. I). Therefore, primary hyperparathyroidism was excluded. Renal and thyroid function was normal. Her recent oncological assessment (three months earlier) showed complete remission of ovarian cancer. Thus, diagnosis of bone metastases was unlikely. On densitometry T-score in the lumbar spine and in the femoral neck were (-)1.3 and $(-) 0.6$, respectively. A bone scintiscan indicated increased tracer uptake in both parietal and zygomatic bones, jaws, and mandible as well as in the shafts of both tibia bones, suggesting focally increased bone metabolism (Fig. 1A). Her scull on X-ray examination presented diffused poorly demarcated sclerotic changes with slight thickening of the frontal bone. On X-ray of both tibiae no visible changes were detected. She was eventually diagnosed with Paget's disease and treated with orally administrated alendronate ( $40 \mathrm{mg} /$ week) with calcium supplementation (1000 mg/day) and cholecalciferol (4000 IU/day) for six months. The therapy resulted in a significant improvement of symptoms; the bone pain in the scull and tibiae subsided. Blood tests following six-month therapy (Tab. I) revealed normalisation of parathormone level $(50 \mathrm{pg} / \mathrm{mL})$ accompanied by increase in 25-hydroxyvitamin D concentration and decrease in alkaline phosphatase, while serum osteocalcin (measured for the first time) was normal. A repeated bone scintiscan was performed, demonstrating no foci of pathologically increased uptake in the skeleton (Fig. 1B).

\section{Discussion}

Paget's disease (PD) is a focal disorder of bone remodelling that progresses slowly and leads to changes in the shape and size of affected bones. It is the second most common metabolic bone disease following osteoporosis in some parts of the world. It affects $2-4 \%$ of adults over 55 years of age. The disease can be quite easily diagnosed and effectively treated, but its pathogenesis remains incompletely understood [1].

There is a slight male preponderance in PD incidence. Affected bone becomes enlarged and mechanically weakened, leading to pain, skeletal deformities, and fractures. However, most PD patients are asymptomatic and diagnosed because of increased alkaline phosphatase activity or incidental X-ray findings. Most commonly the disease involves the axial skeleton, particularly the pelvis, lumbosacral spine, skull, or less frequently - femur or tibia. The disease may be limited to one or a few bones or widespread [2].

Clinically PD may present with constant, diffuse or localised pain. Deformities such as bowing of long bones occur due to abnormal distribution of forces. Mechanical pain of the affected limb can arise due to cortical fissuring. The most common neurological complication is deafness arising from compression of the eighth cranial nerve. Other neurological problems include vertigo, spinal cord compression, local compressive syndromes, 

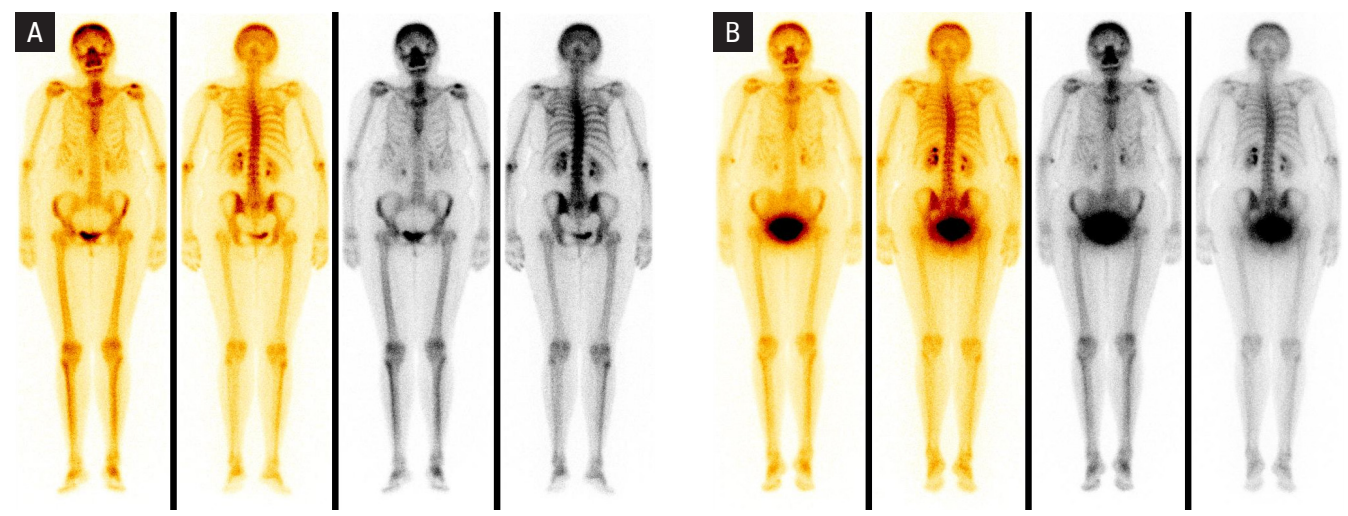

Figure 1. Whole-body bone scintiscan of a patient on diagnosis demonstrating an increased uptake in both parietal and zygomatic bones, jaws, and mandible as well as in the shafts of both tibia bones (A), and six months after oral therapy with alendronate (B)

Table I. Biochemical parameters characterising calcium-phosphate balance and bone metabolism on diagnosis and following alendronate therapy

\begin{tabular}{|c|c|c|c|c|c|}
\hline Parameter & Reference range & Value on diagnosis & Interpretation & Value after therapy & Interpretation \\
\hline lonised calcium [mg/dL] & $4.2-5.2$ & 5.27 & $\uparrow$ & 5.49 & $\uparrow$ \\
\hline Total calcium [mg/dL] & $8.80-10.20$ & 10.11 & $\leftrightarrow$ & 9.95 & $\leftrightarrow$ \\
\hline Phosphate [mg/dL] & $2.7-4.5$ & 4.08 & $\leftrightarrow$ & 3.34 & $\leftrightarrow$ \\
\hline Parathormone $[\mathrm{pg} / \mathrm{mL}]$ & $15-65$ & 73 & $\uparrow$ & 50 & $\leftrightarrow$ \\
\hline $25-\mathrm{OH}$ vitamin $\mathrm{D}[\mathrm{ng} / \mathrm{mL}]$ & $30-80$ & 25 & $\downarrow$ & 60 & $\leftrightarrow$ \\
\hline Alkaline phosphatase $[\mathrm{U} / \mathrm{L}]$ & 35-105 & 156 & $\uparrow$ & 128 & $\uparrow$ \\
\hline Serum osteocalcin [ug/L] & $5-25$ & - & - & 21 & $\leftrightarrow$ \\
\hline Calcinuria [mg/24h] & $100-320$ & 224 & $\leftrightarrow$ & 233 & $\leftrightarrow$ \\
\hline
\end{tabular}

other cranial nerve palsies, hydrocephalus, and brain stem compression from basilar invagination [3].

The diagnosis of PD can be made on the basis of increased markers of bone remodelling, accompanied by focally increased tracer uptake on bone scintiscan. The first and only sign of PD is often elevated ALP level. Isotope bone scanning is the best method of detecting the extent of skeletal involvement [4]. Pagetic lesions may be more easily detected by bone scintigraphy than conventional radiology, due to its higher sensitivity. Paget disease diagnosis is made by exclusion, taking into consideration vitamin D deficiency, hyperparathyroidism, hyperthyroidism, renal osteodystrophy, malignancy, fibrous dysplasia, chronic osteomyelitis, and bone metastases $[3,5]$.

Bisphosphonate therapy is indicated for patients with symptomatic disease and should also be considered in patients in whom localisation of affected bones implies the risk of complications. The treatment of choice is a single infusion of zoledronic acid; however, literature data and our case demonstrate that courses of oral alendronate or risedronate are also effective [1]. Besides osteoporosis, alendronate is also FDA-approved for the treatment of PD. Our report demonstrates that oral therapy with alendronate administered once a week might be an effective and well-tolerated option for patients with PD with less severe changes.

\section{Funding}

None.

\section{Conflict of interest}

None.

\section{Authorship}

All authors had access to the data and a role in writing this manuscript.

\section{References}

1. Appelman-Dijkstra NM, Papapoulos SE. Paget's disease of bone. Best Pract Res Clin Endocrinol Metab. 2018; 32(5): 657-668, doi: 10.1016/j. beem.2018.05.005, indexed in Pubmed: 30449547.

2. Kumar AA, Kumar Pg, Prakash Ms, et al. Paget's disease diagnosed on bone scintigraphy: Case report and literature review. Indian J Nucl Med. 2013; 28(2): 121-123, doi: 10.4103/0972-3919.118258, indexed in Pubmed: 24163523.

3. Senthil V, Balaji S. Monostotic Paget Disease of the Lumbar Vertebrae: A Pathological Mimicker. Neurospine. 2018; 15(2): 182-186, doi: 10.14245/ns.1834922.461, indexed in Pubmed: 29991249.

4. Cyranska-Chyrek E, Szczepanek-Parulska E, Ruchala M. A scintigraphic demonstration of a solitary orbital Paget's disease. Endocrine. 2018; 59(3): 698-699, doi: 10.1007/s12020-017-1500-5, indexed in Pubmed: 29282648.

5. Cyranska-Chyrek E, Szczepanek-Parulska E, Markuszewski J, et al. Sudden Hip Pain in a Young Woman. Am J Med. 2017; 130(9): e379-e381, doi: 10.1016/j.amjmed.2017.05.004, indexed in Pubmed: 28583431. 